\title{
Spazio duale dello spazio delle matrici infinite limitate.
}

Nota di Bruno Pint (a Bologna (*).

Sunto. - Si dà la condizione necessaria e sufficiente cui deve soddisfare la matrice infinita $\mathrm{A} \equiv\left\|\mathrm{ahk}_{\mathrm{k}}\right\|(\mathrm{h}, \mathrm{k}=1,2, \ldots)$ affinchè $\mathrm{A} \cdot \mathrm{X}=\sum_{1}^{\infty} \Sigma_{\mathrm{hk}} a_{\mathrm{hk}} \mathrm{x}_{\mathrm{hk}}$ sia convergente qualunque sia la matrice infinita limitata $\mathrm{X} \equiv\|\mathrm{xhk}\|(\mathrm{h}, \mathrm{k}=1,2, \ldots)$; si stabiliscono poi alcune proprieta di una tale matrice $\mathrm{A}$.

Scopo della presente nota è la ricerca di condizioni per la matrice infinita $\left\|a_{h k}\right\|, h, k=1,2, \ldots$, atte ad assicurare la convergenza della serie doppia $\sum_{h k}^{\infty} a_{h k} x_{h k}$ qualunque sia la matrice infinita $\left\|x_{h k}\right\|, h, k=1,2, \ldots$, limitata completamente continua, $o$, più in generale, soltanto limitata.

Ricordiamo che nell'ordinario spazio hilbertiano numerico reale (i) una matrice infinita $X \equiv\left\|x_{k k}\right\|$ si dice limitata se è la matrice di una sostituzione lineare, ciò̀ tale che il trasformato di un arbitrario $H$-vettore $u$ (ciò un vettore di componenti $u_{1}, u_{2}, \ldots$ con $\Sigma_{1}^{\infty} u_{k}{ }_{k}$ convergente, del quale indiche.

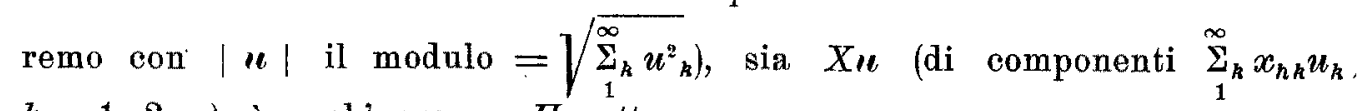
$h=1,2, \ldots$ ), əे anch' esso un $H$-vettore,

Come è noto, una sostituzione lineare trasforma ogni insieme di $H$-vettori di moduli limitati in un insieme di $H$-vettori anch'essi di moduli limitati; in altre parole, a ogni matrice $X$ di una sostituzione lineare si può associare un numero non negativo $M$ per cui $\left|X_{\prime \prime}\right| \leq M|u|$ qualunque sia l'H-vettore $u$.

Indichiamo propriamente con $M_{X}$ (estremo superiore della matrice $X$ ) il più piccolo numero non negativo (nullo solo nel caso che $X$ sia la matrice nulla) per cui sussiste la relazione scritta sopra.

Orbene, la totalità delle matrici limitate si può pensare come uno spazio lineare metrico qualora si facciano le seguenti posizioni:

si definisca come somma di due matrici $X$ e $Y$ la matrice $X+Y \equiv$ $\equiv\left\|x_{h k}+y_{h k}\right\|$

(*) Lavoro eseguito nel Seminario Matematico dell'Università di Bologna.

(1) Per tutti i coneetti e proprieta sui vettori e matrici nello spazio hilbertiano, qui richiamati, rimandiamo a F. RiEsz, Les systèmes d'équations linéaires à une infinité d'inconnues, Paris, 1913; oppure all'articolo di E. HeLLINGgR e O. TofPLITz, in Encyklopädie $d$. Mathem. Wissensch. II Bd. III Teil., 2. 
si definisca come prodotto di una matrice $X$ per un numero reale $\lambda$ la matrice $\lambda X \equiv\left\|\lambda x_{h k}\right\|$;

ad ogni matrice $X$ si associ l' anzidetto numero non negativo $M_{X}$, da consider:re come il suo modulo o norma, per il quale si ha $M_{\lambda X}=|\lambda| M_{X}$, $M_{X+X} \leq M_{X}+M_{Y}$

si definisca come distanza di due matrici $X, Y$, il numero $M_{X-Y}$ $\left(=M_{Y-X}\right)$.

Un sottospazio dello spazio or ora definito è poi quello delle matrici limitate completamente continue. Premesso che una successione di $H$-vettori $\left\{{ }^{(\nu)}\right\}, v=1,2, \ldots$, si dice convergente semplicemente a un $H$-vettore $"$ se esiste un numero positivo $m$ tale che $\left|u^{(v)}\right|<m, v=1,2, \ldots$, e si ha $\lim u_{k}^{(\nu)}=u_{k}$, qualunque sia $k$, e si dice invece convergente fortemente se $\lim \left|w-w^{(\nu)}\right|=0$, ricordiamo che una matrice limitata $X$ si dice comple. $\lim _{x \rightarrow \infty}$

tamente continua se, per ogni successione $\left\{\boldsymbol{u}^{(v)}\right\}$ semplicemente convergente, la successione $\left\{X \mu^{(\nu)}\right\}$ dei trasformati è fortemente convergente.

Cio posto, indichiamo con $\Sigma$ lo spazio delle matrici limitate e con $\mathbf{\Sigma}^{\prime}$ il sottospazio delle matrici limitate completamente continue.

Su $\Sigma$ (e analogamente su $\Sigma^{\prime}$ ) consideriamo la funzione additiva $A \cdot X_{3}$ da intendere come somma della serie $\sum_{1}^{\infty} \alpha_{k k} \alpha_{n_{k}} x_{n k}$, essendo $X$ un punto qualsiasi di $\Sigma\left(\Sigma^{\prime}\right)$ e $A \equiv\left\|\alpha_{t k}\right\|$ una certa matrice infinita per cui l'anzidetta serie riesce convergente qualunque sia $X$; indichiamo poi con $\Sigma^{*}\left(\Sigma^{*}\right)$ la totalità delle matrici $A$ per cui ciò si verifica, totalità che, con una locuzione di di uso cor'ente, diremo spazio duale di $\mathbf{\Sigma}\left(\Sigma^{\prime}\right)$.

Com'è stato dichiarato all'inizio, qui ei proponiamo di individuare gli spazi $\Sigma^{*}$ e $\Sigma^{* \prime}$. Rileviamo che mentre lo spazio $\Sigma$ si puó pensare come il duale dello spazio delle matrici $\left\|x_{h} y_{k}\right\|$ con $x=\left(x_{1}, x_{2}, \ldots\right), y=\left(y_{1}, y_{2}, \ldots\right)$ arbitrari $H$-vettori (secondo la classica teoria delle forme bilineari nello spazio hilbertiano), la questione qui trattata in certo qual modo inverte la ricerca inquantochè si vuole lo spazio duale di $\mathbf{\Sigma}$, di cui quello delle matrici $\left\|x_{h} y_{k}\right\|$ è un sottospazio.

Osserviamo ancora che la questione può essere riguardata dal punto di vista dei fattori di convergenza per successioni doppie inquantochè si cercano condizioni per una successione doppia $\left\{a_{i j}\right\}$ atte ad assicurare la convergenza della serie doppia $\sum_{1}^{\infty} a_{i j} x_{i j}$ per ogni successione doppia $\left\{x_{i, j}\right\}$ per cui $\left\|x_{i j}\right\|$ è una matrice infinita limitata 0 , più in particolare, completamente continua. Così, p. es., nel caso particolarissimo che le $X$ siano tutte matrici diagonali limitate $o$, rispettivamente, completamente continue, tutto si riduce ad esprimere la condizione necessaria e sufficiente per la successione $\left\{a_{i i}\right\}$ affinchè riesca convergente la serie $\sum_{i}^{\infty} a_{i i} x_{i i}$ per ogni successione $\left\{x_{i i}\right\}$ limi- 
tata o, rispettivamente, convergente a zero; e cosi come queste due ultime condizioni non sono l'una più restrittiva dell'altra, vedremo che altrettanto avviene per quelle dette prima relativamente a matrici solo limitate 0 , più in particolare, completamente continue.

Orbene, noi daremo dapprima una condizione solo necessaria affinchè la matrice $A$ appartenga a $\Sigma^{* \prime}$, e quindi anche a $\Sigma^{*}$; una condizione solo sufficiente affinchè $A$ appartenga a $\Sigma^{*}$, e quindi anche a $\Sigma^{*}$; proveremo poi che per una matrice $A$ di $\Sigma^{*}\left(\Xi^{* \prime}\right)$ riesce limitato l'insieme dei valori di $A \cdot X$ per ogni insieme di matrici $X$ di $\Sigma(\Sigma)$ per cui è limitato il corrispondente insieme degli estremi superiori; di qui dedurremo una condizione necessaria $\theta$ sufficiente affinchè $A$ appartenga a $\Sigma^{*}$ o a $\Sigma^{*}$, riconoscendo, in conseguenza, la coinoidenza di $\Sigma^{*}$ con $\Sigma^{*}$. Daremo poi una semplice caratterizzazione intrinseca dei sottospazi delle matrici simmetriche di $\Sigma^{*}$ e di $\Sigma^{* \prime}$ e proveremo inoltre una proprieta di continuità del funzionale $A \cdot X$ per successioni $\left\{X_{y}\right\}$ di matrici di $\Sigma$ o di $\Sigma^{\prime}$, semplicemente convergenti.

Cominciamo con l'osservare che:

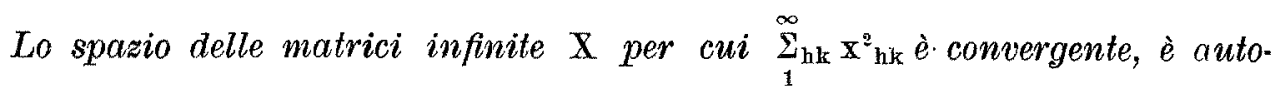
duale, e riesce

$$
|A \cdot X| \leq \sqrt{\sum_{i}^{\infty} x_{h k}^{2}} \sqrt{\sum_{1}^{\infty} a_{h k}^{2}} .
$$

Infatti la serie doppia $\stackrel{\infty}{\Sigma}_{i k}^{\infty} a_{h k} x_{h k}$, se è convergente tutte le volte che $\sum_{1}^{\infty} \sum_{h k} x_{h k}^{2}$ è convergente, allora, oltre che convergente, dovrà risultare assolutamente convergente, come si vede alterando opportunamente di segno le quantità $x_{h k}$ così da rendere positivi tutti i termini $a_{h k} x_{h k}$. Perciò ogni serie semplice ad essa equivalente riuscirà assolutamente convergente; ma ogni successione semplice equivalente alla successione doppia $\left\{x_{h_{k}}\right\}$ rappresenta un $H$-vettore, onde, per un noto teorema di Hellingen.Tokplitz ("), dovrà essere convergente la serie dei quadrati dei termini di una successione sem. plice equivalente alla successione $\left\{a_{h k}\right\}$, ciò̀ la $\sum_{1}^{\infty}{ }_{h k} a_{h k}^{2}$.

Dalla disuguaglianza di LAGRANGE-CAUCHY segue poi che

$$
\left|\sum_{1}^{\infty} a_{h k} x_{h k}\right| \leq \sqrt{\sum_{1}^{\infty} a_{h k}^{2}} \sqrt{\sum_{1}^{\infty} \sum_{h k} x_{h k}^{2}} .
$$

(') Helinghr u. Tomptitz, Grundlagen für eine Theorie der unendichen Matrizen, "Nachrichten Ges. Wiss. Göttingen », 1906, 351-35ō. 
Di quì, poichè $\mathbf{\Sigma}$ contiene $\Sigma^{\prime}$, che a sua volta contiene le matrici $X$ per cui $\Sigma_{h k}^{\infty} x_{h k}^{z}$ è convergente, segue che $\Sigma^{*}$, e quindi anche $\Sigma^{*}$, sono contenuti nello spazio delle matrici $X$ per cui $\sum_{\Sigma_{k k}}^{\infty} x_{h k}^{2}$ è convergente, onde:

I. - Condizione necessaria affinchè A appartenga a $\Sigma^{* \prime}$ (e quindi anche $a \Sigma^{*}$ ) è che sia convergente la serie doppia $\sum_{\mathrm{hkk}}^{\infty} \mathrm{a}_{\mathrm{hk}}^{*}$.

Di seguito alla condizione necessaria 1 . diamo la seguente condizione sufficiente :

II. Condizione sufficiente affinchè A appartenga a $\Sigma^{*}$ (e quindi a $\Sigma^{* \prime}$ ) è che essa sia il prodotto di due matrici $\left\|\alpha_{\mathrm{hk}}\right\| e\left\|\beta_{\mathrm{hk}}\right\|$ tali che riescano convergenti le serie doppie $\sum_{1}^{\infty} \alpha_{\mathrm{hk}}{ }^{2}{ }_{\mathrm{hk}} e \sum_{1}^{\infty}{ }_{\mathrm{hk}} \beta^{2}{ }_{\mathrm{hk}}$.

Si ha infatti, qualunque sia la matrice limitata $X$ :

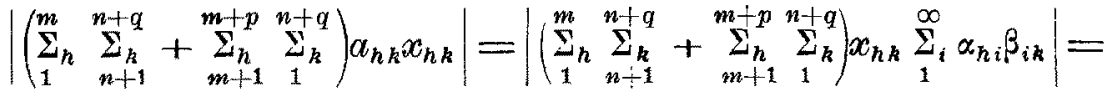

$$
\begin{aligned}
& =\left|\sum_{i}^{\infty}\left(\sum_{1}^{m} \sum_{n+1}^{n+q}+\sum_{m+1}^{m+p} \sum_{1}^{n+q} \sum_{k}\right) x_{h k} \alpha_{n i} \beta_{i k}\right| \leq
\end{aligned}
$$

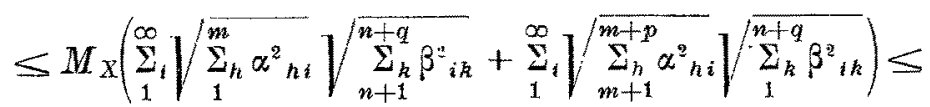

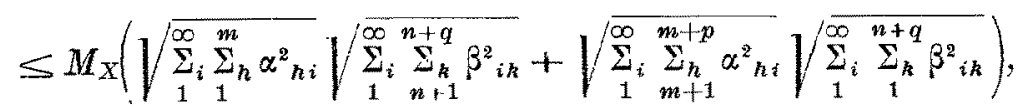

tenendo presente la formula di maggiorazione del modulo di una forma bilineare nello spazio hilbertiano e applicando la disuguaglianza di LAGRANGECAUohy. Ora l' ultimo membro si può rendere minore di un arbitrario numero positivo $\varepsilon$ non appena $m$ ed $n$ siano abbastanza grandi e qualunque siano $p$ e $q$, se si tien presente l' ipotesi.

Si ha poi, sempre nelle ipotesi specificate in II,

$$
|A \cdot X| \leq M_{X} \sqrt{\sum_{1}^{\infty} \sum_{h k} \alpha_{h k}^{2}} \sqrt{\sum_{1}^{\infty} \beta_{h k} \beta_{h k}} .
$$

Osservaztone. - 亡̀ ben noto dalla teoria delle forme bilineari nell, spazio hilbertiano che la condizione necessaria e sufficiente affinchè la matrice infinita $X$ sia limitata è che per ogni coppia di $H$-vettori $u=\left(u_{1}, u_{2} \ldots\right)$ e $v \equiv\left(v_{1}, v_{2}, \ldots\right)$ sia convergente la serie doppia $\sum_{1}^{\infty} x_{h k} u_{h} v_{h}$. Questa condizione è equivalente alla seguente: Condizione necessaria e sufficiente affinchè la matrice $\mathrm{X}$ sia limitata è che sia convergente la serie doppia ${\underset{1}{\sum_{\mathrm{hk}}} \mathrm{x}_{\mathrm{hk}} \mathrm{a}_{\mathrm{hk}}}_{k}$ per ogni matrice $\left\|\mathrm{a}_{\mathrm{bk}}\right\|$ che sia il prodolto di due matrici ciascuna con la serie doppia dei quadrati dei termini convergente. 
Infatti se $X$ è limitata, allora $\sum_{1}^{\infty} x_{h k} x_{h k} \alpha_{h k}$ è convergente per II; se poi $\sum_{\Sigma_{h k}}^{\infty} x_{h k} a_{h k}$ è convergente per ogni matrice $\left\|a_{h k}\right\|$ del tipo sopra detto, lo sarà anche se tale matrice è, più in particolare, del tipo

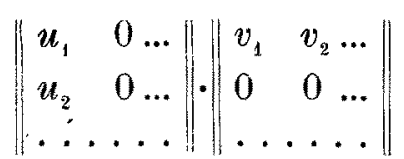

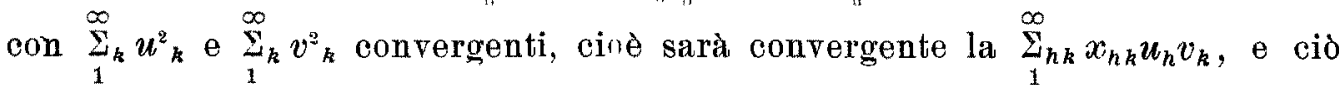
richiede ehe $X$ sia limitata.

Pertanto, se la serie ${\stackrel{\infty}{\Sigma_{k \xi}}}_{1} x_{k k} a_{h k}$ riesce convergente qualunque sia la ma. trice $\left\|a_{k \hbar}\right\|$ prodotto di due matrici $\left\|\alpha_{h k}\right\|,\left\|\beta_{h k}\right\|$, ciascuna delle quali con la serie doppie deí quadrati dei termini convergente, varrà la (1) ed $M_{X}$ sarà il più piccolo numero non negativo $M$ tale che

$$
\left|\sum_{h k}^{\infty} x_{h k} a_{h k}\right| \leq M \sqrt{\sum_{h k}^{\infty} \alpha_{h k}^{2}} \sqrt{\sum_{1}^{\infty}{ }_{h k} \beta_{h k}^{2}},
$$

se si pensa che $M_{X}$ ̀̀ il più piccolo numero non negativo $M$ per oui si ha

$$
\left|{\stackrel{\infty}{\Sigma_{h k}}}_{1}^{\infty} x_{h k} u_{h} v_{k}\right| \leq M \sqrt{\sum_{1}^{\infty} u_{h}^{2}} \sqrt{\sum_{1}^{\infty} v_{k}^{*}}
$$

qualunque siano gli $H$-rettori $", c$, e pensando questi ultimi come particolari matrici $\left\|\alpha_{h k}\right\|$ e $\left\|\beta_{h k}\right\|$ come si è fatto sopra.

Proviamo ora che:

III. - Se A appartiene a $\Sigma^{* \prime}$, esiste un numero positivo $M$ tale che $|A \cdot X|<M M_{X}$, qualunque sia la matrice $\mathrm{X} d i \Sigma^{\prime}$.

Imitando la dimostrazione di un analogo teorema di HeLLINGER-ToEPLITZ $\left({ }^{3}\right)$, ci fonderemo su an certo dilemma.

a) Facciamo dapprima le seguenti ipotesi :

Esistano un intero positivo $\bar{n}$, due numeri positivi $\bar{M}$ e $c$ ed $\overline{n^{\prime}}$ numeri $\bar{x}_{1,1}, \ldots, \bar{x}_{n}^{-}, \bar{n}$, tali che per ogni $n>\bar{n}$ e per ogni sistema di $n^{2}-\bar{n}^{2}$ quantita $x_{1, \bar{n}+1}, \ldots, x_{n, n}$ per cui sia

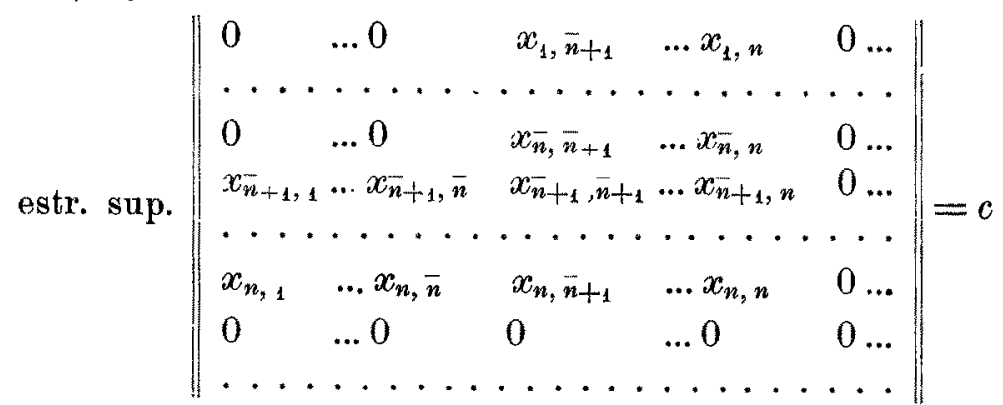

(3) Hellinger u. Toeplttz, Grundlagen für eine Theorie der unendlichen Matrizen, "Math. Annalen ", t. LXIX, 289.330. 
risulti

$$
\left|\sum_{1}^{\bar{n}}{ }_{h k} a_{h k} \bar{x}_{h k}+\sum_{1}^{\bar{n}} \sum_{\bar{n}+1}^{n} a_{h k} x_{h k}+\sum_{\bar{n}+1}^{n} \sum_{1}^{\bar{n}} a_{h k} a_{h k} x_{h+1}+\sum_{\bar{n}+1}^{n} a_{h k} x_{h k}\right|<\bar{M} .
$$

Sarà allora

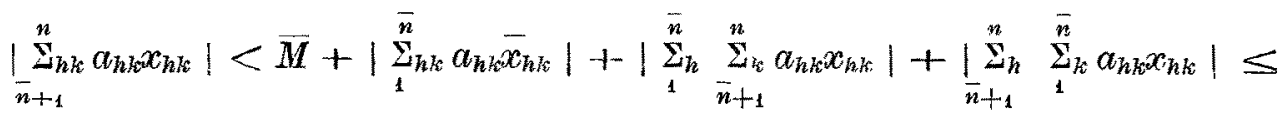

$$
\begin{aligned}
& \leq \bar{M}+\left|\sum_{1}^{\bar{n}} \sum_{h k} a_{h k} \bar{x}_{h k l}\right|+\bar{n} \sqrt{\sum_{1}^{n}\left(\sum_{\bar{n}+1}^{n} a_{h k} x_{h k}\right)^{2}}+\bar{n} \sqrt{\sqrt{\sum_{i}\left(\sum_{n+1}^{n} \sum_{h} a_{h k} x_{k k}\right)^{2}}}
\end{aligned}
$$

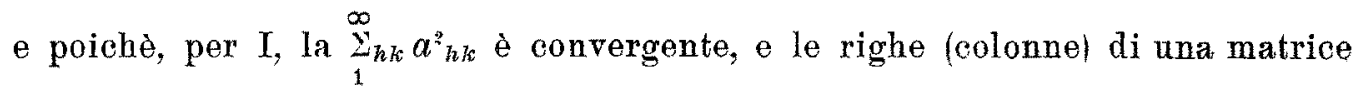
limitata $X$ sono $H$-vettori tutti di modulo non superiore a $M_{X}$, si ha

$$
\left|\underset{\bar{n}+1}{\sum_{h k}^{n}} a_{h k} x_{h k}\right|<\left(\bar{M}+\left|\sum_{1}^{\vec{n}} \sum_{h k} a_{h k} \bar{x}_{h k}\right|+2 \bar{n} c \sqrt{\sum_{i}^{\infty} \alpha_{h k}^{2} \alpha_{h k}}\right) \frac{M_{X}}{c}
$$

ove $M_{X}=c$, indicando con $X$ la matrice di cui in (2).

Sia ora $X$ un'arbitraria matrice limitata completamente contiuna; indicando con $X_{1}$ la matrice da essa ottenuta sostituendo con degli zeri glj elementi comuni alle prime $\bar{n}$ righe ed $\bar{n}$ colonne e tutti i termini con almeno uno degli indici superiore ad $n$, si otterrà una matrice che, moltiplicata per $\frac{c}{M_{X_{1}}}$, soddisferà la condizione (2). Perciò la (3) continua a valere qualunque sia la matrice $X_{1}$, qualunque sia il suo estremo superiore $M_{X_{1}}$. Quindi passando al $\lim _{n \rightarrow \infty}$, tenendo presente che $M_{X_{1}} \leq 2 M_{X}$, si ha

$$
\left|\sum_{\bar{n}+1}^{\infty} a_{h k} x_{h k}\right| \leq 2\left(\frac{\bar{M}}{c}+\frac{1}{c}\left|\sum_{1}^{\bar{n}} a_{h k} \bar{x}_{h k}\right|+2 \bar{n} \mid / \sum_{1}^{\infty} \infty_{h k} a_{h k}^{2}\right) M_{X}
$$

qualunque sia la matrice completamente continua $X$. D'altra parte si ha

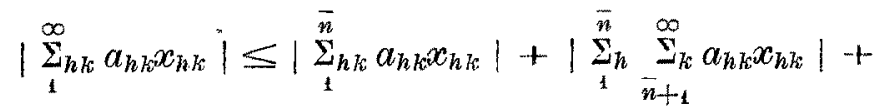

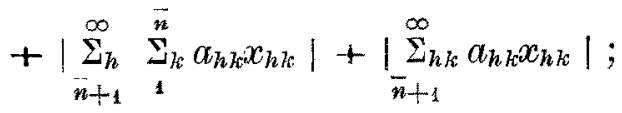

il primo termine a secondo membro viene maggiorato da $\bar{n} M_{X} \sqrt{\sum_{h_{k}}^{\infty} a_{h k}^{2}}$; il secondo e il terzo analogamente; il quarto dalla (4), onde

$$
\left|\sum_{l}^{\infty} a_{h k} x_{h k}\right|<\left(\frac{2 \bar{M}}{c}+\frac{2}{c}\left|\sum_{l}^{\Sigma_{h k}} a_{h k} \bar{x}_{h k}\right|+7 n \sqrt{\sum_{1}^{\infty}} \overline{\sum_{h k} a_{h k}^{2}}\right) \mathbf{M}_{X}
$$


e quindi, detto $M$ il numero $\frac{2 \bar{M}}{c}+\frac{2}{c}\left|\sum_{1}^{\bar{n}} a_{h k} \bar{x}_{h k}\right|+7 \bar{n} \sqrt{\sum_{1}^{\infty} a_{h k} a_{h k}}$, si ha, qualunque sia la matrice limitata completamente continua $X$ :

$$
\left|\sum_{1}^{\infty} a_{h k} a_{h k} x_{h k}\right|<M M_{X} .
$$

b) Poniamoci ora nell'ipotesi contraria :

Qualunque siano l'intero positivo $\bar{n}$, i numeri $\bar{M}$ e $c$ e gli $\bar{n}^{2}$ numeri $x_{i, 1}, \ldots, x_{n}, \bar{n}$, si possano sempre assegnare in corrispondenza un intero $n>\bar{n}$ e un sistema di $n^{2}-\bar{n}^{2}$ quantità $x_{1}, \bar{n}+1, \ldots, x_{n, n}$ per cui valga la (2) e risulti

$$
\left|\sum_{k=}^{n} a_{h k} x_{h k}\right|>\bar{M}
$$

Si fissi allora nna successione divergente di numeri positivi $M_{1}, M_{2}, \ldots$, $M_{\nu}, \ldots$ e una serie a termini positivi convergente $c_{\ell}+c_{2}+\ldots+c_{v}+\ldots$. Fissato a piacere l'intero positivo $n_{0}$ e gli $n_{0}^{2}$ numeri $x_{1,1}, \ldots, x_{n_{0}, n_{0}}$, vi saranno un intero $n_{1}>n_{0}$, e un sistema di $n_{1}^{2}-n_{0}^{2}$ quantità $x_{1, n_{0}+1}, \ldots, x_{n_{1}, n_{1}}$ per cui

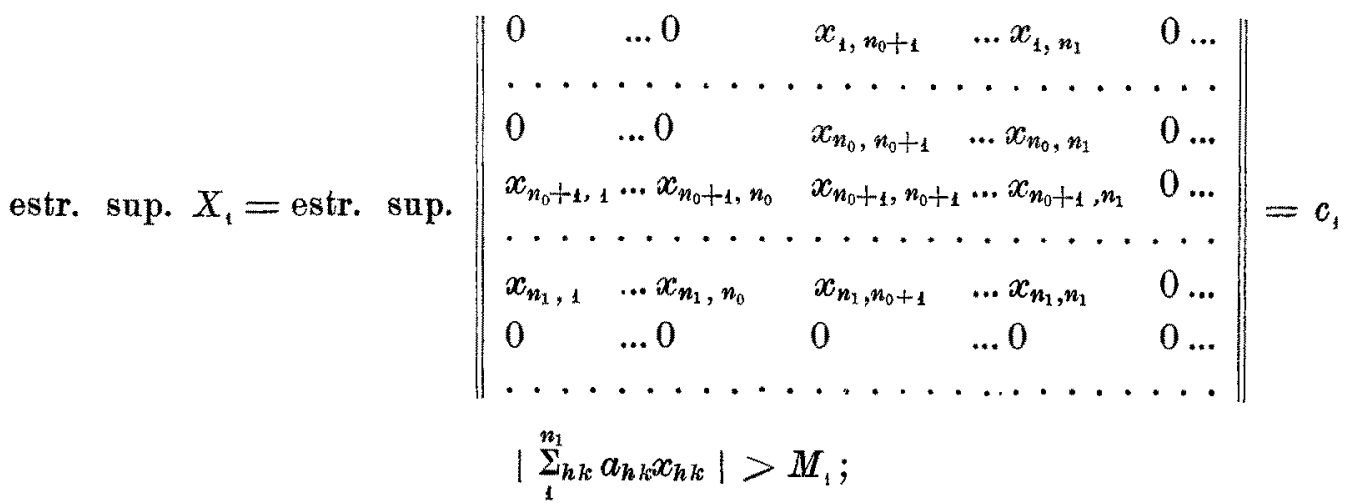

poi un intero $n_{2}>n_{1}$ e un sistema di $n_{2}^{2}-n_{1}^{2}$ quantítà $x_{1, n_{1+1}}, \ldots, x_{n_{2}, n_{2}}$ con

estr. sup. $X_{2}=$ estr. sup.

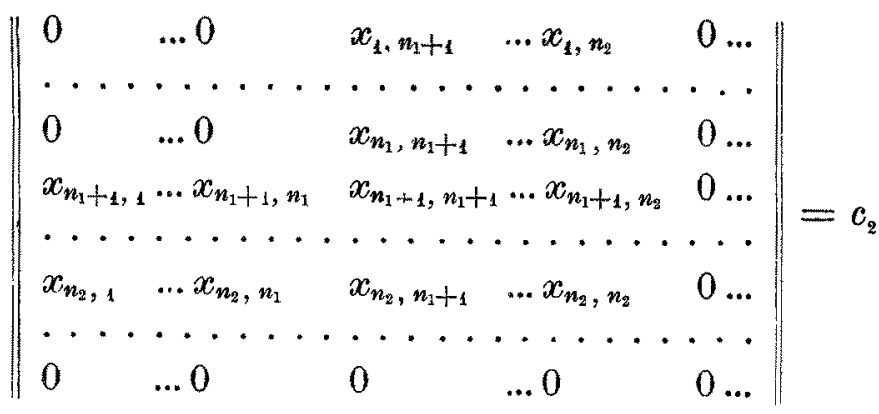

$$
\begin{aligned}
& \left|\sum_{1}^{n_{2}} a_{h k} x_{h k}\right|>M_{2} ; \text { ecc. }
\end{aligned}
$$


Si viene cosi a definire una successione crescente di numeri naturali $n_{0}, n_{1}, \ldots, n_{v}, \ldots$ e una doppia successione di numeri $\left\{x_{k k}\right\}$ costituenti una matrice limitata eompletamente continua; infatti le ridotte della serie $X_{1}+X_{2}+\ldots+X_{y}+\ldots$, convergono fortemente $\left(^{4}\right)$ verso la matrice $X \equiv\left\|x_{h k}\right\|$ poichè

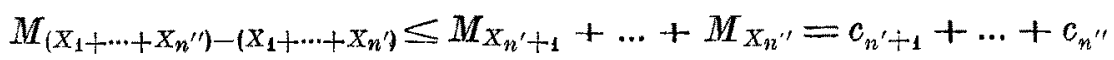

che per $n^{\prime}$ maggiore di un certo intero $n_{\varepsilon}$ e qualunque sia $n^{\prime \prime}>n^{\prime}$, riesce minore di un prefissato $\varepsilon>0$ arbitrario. Onde, tenendo presente che ogni successione fortemente convergente di matrici limitate completamente continue converge a una matrice limitata completamente continua, si può concludere che $X$ ̀े completamente continua. Ora, qualunque sia l'intero positivo $v$, riesce

$$
\left|\Sigma_{i}^{n_{y}} a_{h k} x_{h k}\right|>M_{y}
$$

onde la serie $\sum_{1}^{\infty} a_{h k} a_{h k} x_{h k}$ ba la successione delle ridotte quadrate non limitata e quindi non può essere convergente, contro l'ipotesi.

In modo perfettamente simile si prova che:

III'. Se A appartiene a $\Sigma^{*}$, esiste un numero positivo $\mathrm{M}$ tale che $|\mathrm{A} \cdot \mathrm{X}|<\mathrm{MM}_{\mathrm{X}}$ qualunque sia la matrice $\mathrm{X}$ di $\mathbf{\Sigma}$.

Basta, nella prima parte della dimostrazione di III, parlare di matriei limitate anzichè di matrici limitate completamente continue.

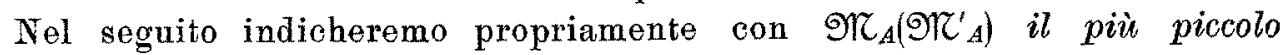
numero non negativo per cui si ha

$$
|A \cdot X| \leq \mathfrak{N}_{A} M_{X} \quad\left(\mathfrak{O K}_{A} M_{X}\right)
$$

riferendoci a III (III').

A questo proposito osserviamo che:

IV. Se A appartiene a $\Sigma^{*}$, e quindi anche a $\Sigma^{* \prime}$, riesce $\mathfrak{O K}_{\mathrm{A}}=\mathfrak{K}_{\mathrm{A}}^{\prime}$.

Infatti, cominciamo con l'osservare che dalla sola ipotesi che sia convergente la serie doppia $\sum_{1}^{\infty} a_{h k} x_{h k}$ per ogni matrice limitata $X$, segue che essa sarà sommabile tanto per righe quanto per colonne (poichè ciascuna sua riga, e colonna, è convergente). Non solo, ma la sommabilità per righe e per colonne sarà assoluta, cioè riusciranno convergenti le serie $\sum_{1}^{\infty}\left|\sum_{1}^{\infty} a_{h k} x_{h k}\right|$ e $\sum_{i}^{\infty}\left|\Sigma_{1}^{\infty} a_{h k_{0} x_{h l}}\right|$; basta infatti tener presente che se $X$ ஓे una matrice limi-

(4) Rícordiamo che una successione $X^{(v)}$, di matrici limitate si dice convergente sem. plicemente alla matrice $X$ se esiste un numero positivo $m$ tale che $M_{X(v)}<m$ per $v=1,2, \ldots$, e lim $x_{h k}^{()}=x_{h k}$ per ogni coppia $h, k=1,2, \ldots$; si dice invece fortemente convergente se

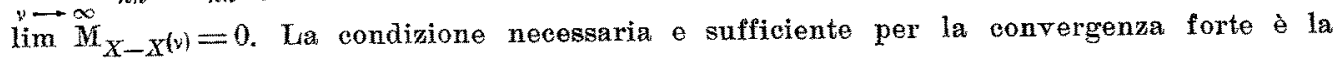
condizione di CAUCHX applicata nel testo. 
tata, tale è anche quella che si ottiene mutando il segno a tutti i termini di quante e quali si vogliano righe o colonne.

Ora è $\mathfrak{T K}_{A} \geq \mathfrak{T K}_{A}^{\prime}$; nell' ipotesi che sussista il segno di disuguaglianza, sia $X$ una matrice limitata, di estremo superiore 1 , per eui si abbia

$$
\left|\sum_{1}^{\infty} a_{h k} x_{h k}\right|=\mathscr{T K}_{A}
$$

fissato a piacere un numero positivo $\varepsilon$, si scelga una successione $\rho_{1}, \rho_{2}, \ldots$ di numeri, ciascuno di modulo non superiore a 1 , convergente a zero, per cui riesca

$$
\left|\sum_{1}^{\infty}\left(p_{h}{\stackrel{\infty}{\Sigma_{k}}}_{1}^{\infty} \alpha_{h k} x_{h k}\right)\right|>\mathfrak{K}_{A}-\varepsilon
$$

che tale scelta sia possibile è chiaro per quanto si è detto sopra perchè, riuscendo

$$
\underset{n+1}{\infty}\left|\sum_{1}^{\infty} a_{h k} x_{h k}\right|<\varepsilon
$$

per $n$ abbastanza grande, sarz̀ anche $|\sum_{n+1}^{\infty} \sum_{h} \overbrace{1}^{\infty} \Sigma_{k} a_{h k} x_{h k}|<\varepsilon$, qualunque siano i $\rho_{n+1}, \rho_{n+2}, \ldots$ ciascuno in modulo non superiore a uno, onde, prendendo p. es. $\rho_{1}=\rho_{z}=\ldots=\rho_{n}=1$, sarà

$$
\left|\sum_{1}^{\infty} a_{h k \rho_{h} x_{h k}}\right|>\mathfrak{N K}_{A}-\varepsilon .
$$

Ma la matrice $\left\|\begin{array}{cccc}\rho_{1} & 0 & 0 & \ldots \\ 0 & \rho_{2} & 0 & \ldots \\ 0 & 0 & \rho_{3} & \ldots \\ \ldots & \ldots & \ldots\end{array}\right\|$ è completamente continua se $\lim _{h \rightarrow \infty} \rho_{h}=0$, e quindi anche la matrice $\left\|\rho_{h} x_{h k}\right\|$ è completamente continua, onde, per III,

$$
\left|\sum_{1}^{\infty} a_{h k \rho} x_{h k}\right| \leq \mathscr{T R}_{A}^{\prime} M_{\left\|l_{k} x_{k k}\right\|}<\operatorname{Or}_{A}^{\prime}
$$

e quindi $\mathfrak{N}_{A}-\mathfrak{N}_{A}^{\prime}<\varepsilon$ ehe, per l'arbitrarieta di $\varepsilon$, richiede che sia $\mathfrak{O K}_{A}=\mathfrak{M K}_{A}^{\prime}$.

Da III e III' possiamo dedurre che:

V. Condizione necessaria affinchè A appartenga a $\Sigma^{*}$ (e quindi a $\Sigma^{*}$ )

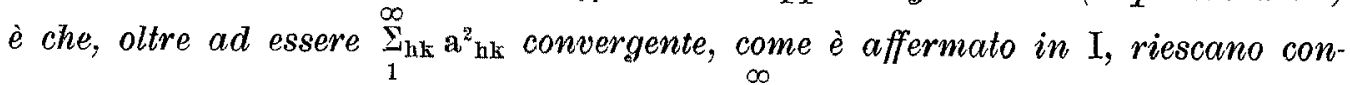
vergenti, con somme limitate, tulte le serie $\sum_{1}^{\infty}\left|a_{\mathrm{ih}_{\mathrm{i}}}\right|$ ove la successione $\left\{\mathrm{h}_{\mathrm{i}}\right\}$ si oltiene permutando in modo arbitrario i termini della successione dei numeri naturali o di una qualunque sua subordinata. 
L'affermazione segue subito la III se, come matrice $X$, si prende una matrice dedotta dalla matrice unita permutando in modo arbitrario le colonne dopo averne eventualmente mutato di segno tutti o parte dei termini. Con ció, veramente, si ha una condizione necessaria affinchè $A$ appartenga a $\Sigma^{*}$; ma la stessa condizione è necessaria affinchè $A$ appartenga a $\Sigma^{* \prime}$. Sia infatti $\alpha_{1}+\alpha_{2}+\ldots$ una delle serie di cui è detto nell' enunciato; supponiamo che la $\stackrel{\infty}{1}_{1}^{\infty}\left|\alpha_{k}\right|$ sia divergente; si potrà allora trovare una successione $n_{n_{y}}, n_{2}, \ldots$ $n_{\nu}, \ldots$ di interi crescenti in modo che sia $\underset{n_{y-1}+1}{\sum_{y}}\left|\alpha_{k}\right|>1$; consideriamo la successione $\rho_{k}=\operatorname{segn} . \alpha_{k} \cdot \frac{1}{v}$ per $k=n_{\nu-1}+1, \ldots, n_{y}\left(n_{3}=0\right), \nu=1,2, \ldots ; 1$ a matrice che ha tritti i termini nulli ad eccezione degli omologhi degli $\alpha_{k}$ di $A$, rispettivamente eguali ai $\rho_{k}$, è completamente continua; detta $R$ tale matrice, riesce $\sum_{1}^{\infty} \sum_{h k} a_{h k} r_{h k}=\sum_{1}^{\infty}\left|\alpha_{k}\right| \cdot\left|\rho_{k}\right|=\sum_{1}^{\infty}\left(\sum_{n_{y-1}+1}^{n_{y}}\left|\alpha_{k}\right|\right) \frac{1}{v}$, divergente perchè maggionante della serie $\sum_{1}^{\infty} \frac{1}{v}$.

Da III si ha inoltre che :

VI. Condizione necessaria e sufficiente affinchè A appartenga a $\Sigma^{*} \grave{e}$ che esista un numero $\mathfrak{\Upsilon R}_{\mathrm{A}}$ tale che, qualunque siano la coppia di interi posi. tivi $\mathrm{m}, \mathrm{n}$ e gli mn numeri $\mathrm{x}_{\mathrm{hk}}, \mathrm{h}=1,2, \ldots, \mathrm{m} ; \mathrm{k}=1,2, \ldots, \mathrm{n}$, sia

$$
\left|\sum_{1}^{m} \sum_{1}^{n} \sum_{k} a_{h k} x_{h k}\right| \leq \mathfrak{O K}_{A} M_{X}
$$

intendendo con $\mathrm{M}_{\mathrm{X}}$ l'estremo superiore della matrice $\left\|\mathrm{x}_{\mathrm{bl}}\right\|, \mathrm{h}=1,2, \ldots, \mathrm{m}$; $\mathrm{k}=1,2, \ldots, \mathrm{n}$.

La necessità della condizione si deduce da III usando una matrice (limitata) avente tutti nulli gli elementi col primo indice $>m$ e quelli col secondo indice $>n$ (che è ovviamente completamente continua). La condizione è anche sufficiente; infatti si ha, indicando con $\vee$ un numero naturale $>m, n$ :

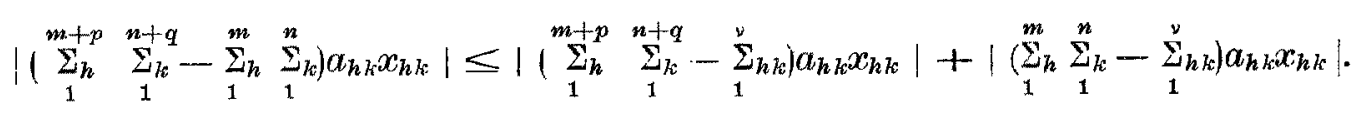

Ora è, p. es.,

$$
\begin{aligned}
& \left.\mid \underset{1}{\left(\Sigma_{h}^{m}\right.} \Sigma_{k}^{n}-\Sigma_{1}^{\nu} \Sigma_{h k}\right) a_{h k} x_{h k}|\leq| \underset{1}{\sum_{h}^{\nu}} \sum_{\nu+1}^{n} a_{h k} x_{h k}|+| \underset{\nu+1}{\Sigma_{h}^{m}} \stackrel{\nu}{\Sigma}_{k}^{\nu} a_{h k} x_{h k} \mid+ \\
& +\left|\sum_{y+1}^{m} \sum_{i+1}^{n} a_{h k} x_{h k}\right| \leq \operatorname{NC}_{A}\left(M_{P_{v}}+M_{Q_{v}}+M_{R_{v}}\right)
\end{aligned}
$$


indicando con $P_{\nu}, Q_{\nu}, R_{v}$ le matrici ottenute da una matrice limitata completamente continua $X$ sostituendo con lo zero gli elementi $x_{h k}$ di questa per

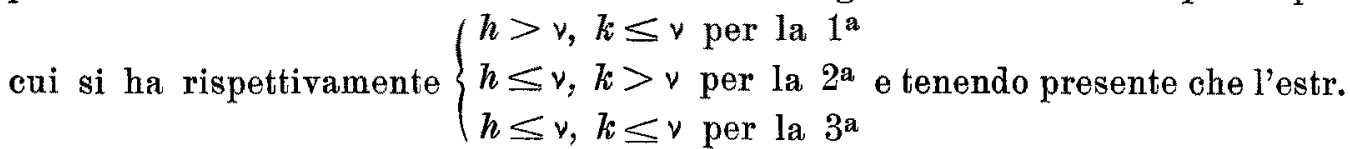
sup. di una matrice limitata non cresce se in essa si sostituiscono con degli zeri tutti gli elementi di una riga (colonna). Ora è noto $\left(^{5}\right)$ che se $X \dot{\text { com- }}$ pletamente continua si ha $\lim \left(M_{P_{v}}+M_{Q_{y}}+M_{R_{v}}\right)=0$, onde, scelto a piacere un $\varepsilon>0$, si può trovare un intero positivo $\vee$ tale che per ogni coppia di interi positivi $m, n$, entrambi maggiori di $v$, e qualunque siano gl'interi non negativi $p$ eq, sia

$$
\left|\left(\sum_{1}^{m+p} \sum_{1}^{n+q} \sum_{k}^{n+q}-\sum_{1}^{m} \sum_{1}^{n} \sum_{k}\right)\right| a_{h k} x_{h i c} \mid<\varepsilon
$$

da cui segue l'asserto

VI' Condizione necessaria e sufficiente affinchè A appartenga a $\Sigma^{*} \grave{e}$ che esista un numero ${ }^{\circ} \mathrm{K}_{\mathrm{A}}$ tale che qualunque siano la coppia di interi positivi $\mathrm{m}, \mathrm{n}$ e gli $\mathrm{mn}$ numeri $\mathrm{x}_{\mathrm{hk}}, \mathrm{h}=1,2, \ldots, \mathrm{m} ; \mathrm{k}=1,2, \ldots, \mathrm{n}$, sia

$$
\left|\sum_{1}^{m} \sum_{1}^{m} \sum_{k}^{n} a_{h k} x_{h k}\right| \leq \mathfrak{M}_{A} M_{X}
$$

intendendo con $\mathrm{M}_{\mathrm{X}}$ l'estremo superiore della matrice $\left\|\dot{\mathrm{x}}_{\mathrm{k \textrm {k }}}\right\|, \mathrm{h}=1,2, \ldots, \mathrm{m}$; $\mathbf{k}=1,2, \ldots, \mathbf{n}$.

La necessità della condizione è contenuta in VI. Per quanto riguarda la sufficienza, cominciamo col provare che se $\left\|\mathrm{x}_{\mathrm{hk}}\right\| \dot{e}$ una matrice limitata, se $\mathrm{m}_{1}, \mathrm{~m}_{2}, \ldots ; \mathrm{n}_{1}, \mathrm{n}_{2}, \ldots$ sono due successioni crescenti di numeri naturali

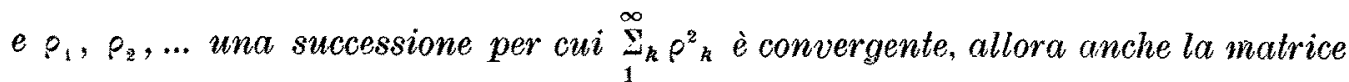

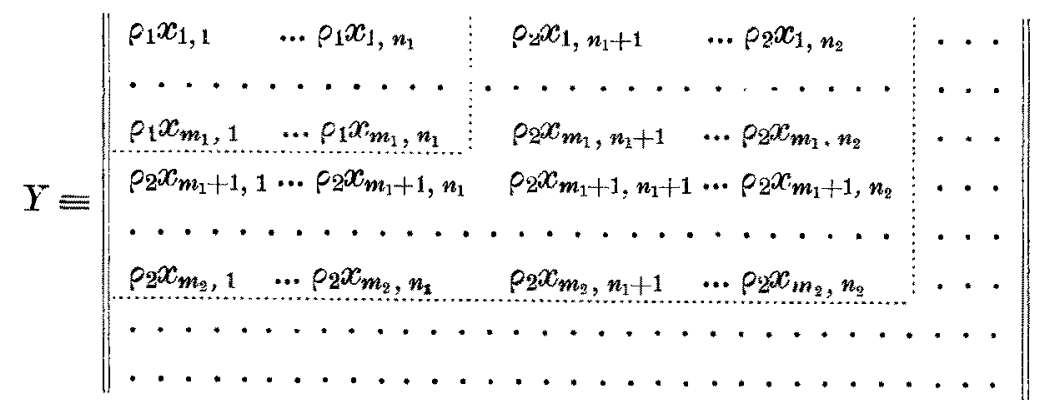

$\grave{e}$ limitata, anzi, di più, completamente continua. Per provare l'asserto mostriamo che è fortemente convergente la successione di matrici completamente

(5) Cfr. p. es. F. Riesz, 1. c. in (1), n. 67 . 
continue

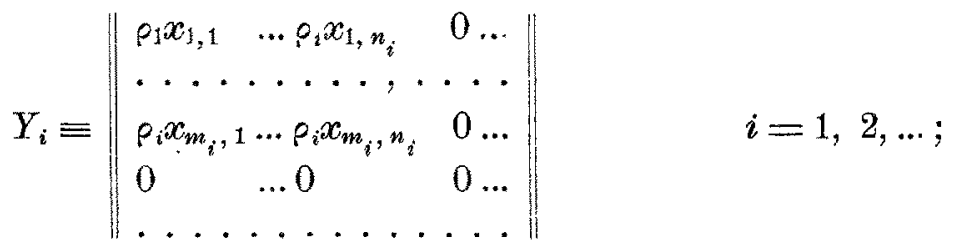

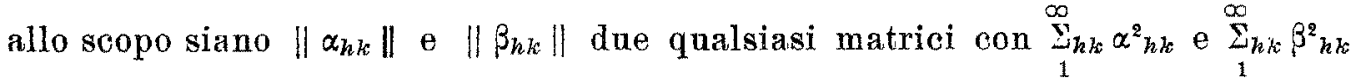
convergenti, di prodotto $Q \equiv\left\|\omega_{h k}\right\|$; si ha

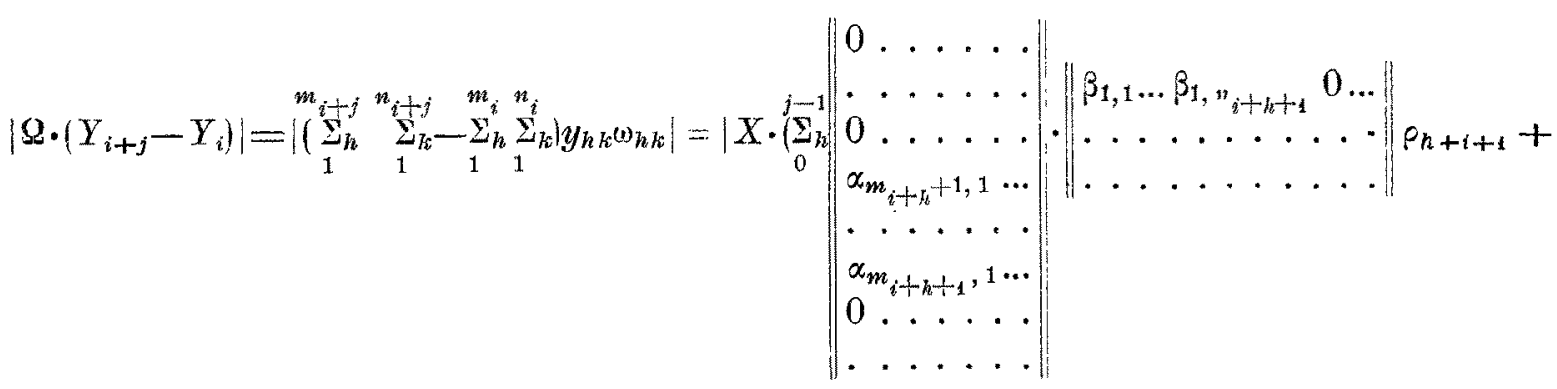

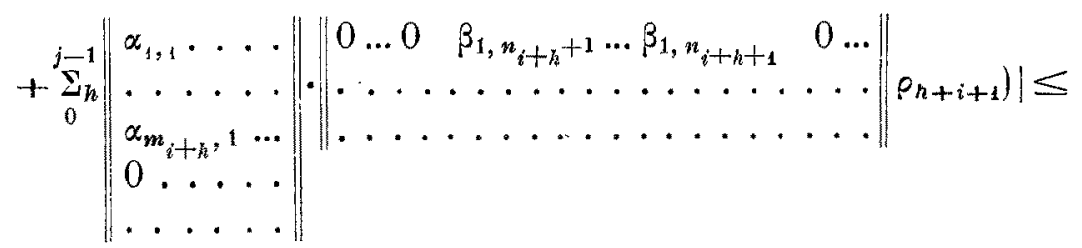

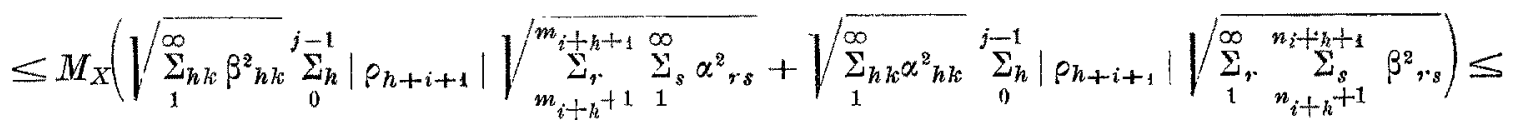

$$
\begin{aligned}
& \leq 2 M_{X} \sqrt{\sum_{0}^{1} \rho_{h+i+1}^{1}} \sqrt{\sum_{1}^{\infty} \rho_{h h} \alpha_{h k}^{2}} \sqrt{\sum_{1}^{\infty} \sum_{h k} \beta_{h k}^{2}}
\end{aligned}
$$

tenendo presente che

$\sqrt{\sum_{m_{i}+1}^{m_{i+1}} \sum_{1}^{\infty} \alpha_{, s}^{2}} i=0,1, \ldots \quad\left(m_{0}=0\right) \quad$ e $\sqrt{\sum_{1}^{\infty}{ }_{n_{i}+1}^{\infty} \sum_{s}^{n_{i+1}} \beta^{2}{ }_{r s}} \quad i=0,1, \ldots \quad\left(n_{0}=0\right)$

sono le componenti di due $H$-vettori, che, per ipotesi, $\sum_{1}^{\infty} \rho_{k}^{2} \dot{\theta}$ convergente, e applicando il teorema di HeLuINGER-ToEPLITz richiamato in ( $\left.{ }^{2}\right)$; onde, per l'osservazione fatta in II, riesce

$$
M_{Y_{i+j}-Y_{i}} \leq 2 M_{X} \sqrt{\sum_{0}^{j-1} \rho^{2}{ }_{i+h+1}}
$$

quantità che sarà $<\varepsilon$, positivo fissato a piacere, non appena $i$ sia abbastanza grande e qualunque sia $j$. Segue che la successione $\left\{Y_{i}\right\}$ converge fortemente e quindi $Y$ è una matrice limitata, anzi completamente continua. 
Ciô premesso, ritornando a quanto volevamo provare all'inizio, suppo. niamo, per assurdo, che esista una matrice limitata $X$ tale che ${\stackrel{\infty}{\Sigma_{h k}}}_{a_{h k} x_{h k}}$ non sia convergente. La condizione enunciata in $\mathrm{VI}^{\prime}$ assicura che tale serie doppia sarà allora oscillante; indichiamo con $\lambda^{\prime \prime} \theta$ con $\lambda^{\prime}$ rispettivamente il max lim e il $\min _{m, n \rightarrow \infty}$ dim della successione doppia delle somme parziali $S_{m, n}$. Possiamo sempre supporre che, per un certo $\delta>0$, sia $\lambda^{\prime}+\delta<0<\lambda^{\prime \prime}-\delta$ /se ciò non fosse, basterebbe aggiungere una apportuna quantità al primo termine della serie doppia in oggetto). Potremo trovare una coppia di successioni crescenti di numeri naturali $m_{1}, m_{2}, \ldots ; n_{1}, n_{2}, \ldots$, in modo che sia

$$
S_{m_{i}, n_{i}} \text { compreso tra }\left\{\begin{array} { l } 
{ \lambda ^ { \prime \prime } - \delta \text { e } \lambda ^ { \prime \prime } + \delta } \\
{ \lambda ^ { \prime } - \delta \text { e } \lambda ^ { \prime } + \delta }
\end{array} \text { rispettivamente per } i \left\{\begin{array}{l}
\text { dispari } \\
\text { pari }
\end{array} .\right.\right.
$$

Indichiamo con $\sigma_{m, n}$ le somme parziali della serie doppia $\stackrel{\infty}{\Sigma}_{\Sigma_{h k}}^{\infty} a_{h k} y_{h k}$; sară

$$
\begin{aligned}
\sigma_{m_{2 k} \cdot n_{2 k}=}=\rho_{1} S_{m_{1}, n_{1}}+\rho_{2}\left(S_{m_{2}, n_{2}}-\right. & \left.S_{m_{1}, n_{1}}\right)+\ldots+\rho_{2 k}\left(S_{m_{2 k}, n_{2 k}}-S_{m_{2 k-1}, n_{2 k-1}}\right)= \\
= & S_{m_{1}, n_{1}}\left(\rho_{1}-\rho_{2}\right)+S_{m_{3}, n_{3}}\left(\rho_{3}-\rho_{4}\right)+\ldots+S_{m_{2 k-3}, n_{2 k-3}\left(\rho_{2 k-3}-\rho_{2 k-2}\right)+} \\
& \quad+S_{m_{2 k-1}, n_{2 k-1}}\left(\rho_{2 k-1}-\rho_{2 k}\right)+ \\
+ & S_{m_{2}, n_{2}}\left(\rho_{2}-\rho_{3}\right)+S_{m_{4}, n_{4}}\left(\rho_{4}-\rho_{5}\right)+\ldots+S_{m_{2 k-2}, n_{2 k-2}\left(\rho_{2 k-2}-\rho_{2 k-1}\right)+}+ \\
& +\rho_{2 k} S_{m_{2 k}, n_{2 k}} .
\end{aligned}
$$

Scegliamo ora $i \rho$ in modo che sia $\rho_{h}>0$ per $h$ dispari $<\sum_{1}^{\infty}\left|\rho_{h}\right|$ ser $h$ pari divergente, ciò ch'è sempre possibile compatibilmente con l'ipotesi che sia $\sum_{1}^{\infty} \rho_{h}^{2}$ convergente. Allora sarà

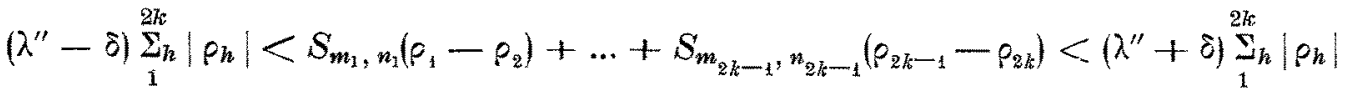

$$
\begin{aligned}
& \left|\lambda^{\prime}+\delta\right| \sum_{2}^{2 k-1}\left|\rho_{h}\right|<S_{m_{2}, n_{2}}\left(\rho_{2}-\rho_{3}\right)+\ldots+S_{m_{2 k-2}, n_{2 k-2}}\left|\rho_{2 k-2}-\rho_{2 k-1}<\right| \lambda^{\prime}-\delta\left|\sum_{2}^{2 k-1}\right| \rho_{h} \mid
\end{aligned}
$$

mentre $\lim _{n \rightarrow \infty} p_{2 k} S_{m_{2 k}, n_{2 k}}=0$. Ma allora, per $k$ abbastanza grande, $\sigma_{m_{2 k}, n_{2 k}}$ prende valori grandi a piacere, ciô che contraddice l'ipotesi di $\mathrm{VI}^{\prime}$ e il fatto, provato sopra, che $Y$ è una matrice limitata.

Pertanto, se si raccolgono i risultati fin qui conseguiti, si può concludere dicendo che :

$\mathrm{VI}^{\prime \prime}$. Gli spazi $\Sigma^{*}$ e $\mathrm{\Sigma}^{* \prime}$ coincidono in un unico spazio lineare metrico al quale appartengono tutte e sole le matrici A che godono della seguente proprietà : a ciascuna di esse si può associare un numero non negativo $\mathscr{T K}_{\mathrm{A}}$ per cui $\left|\stackrel{\infty}{\mathrm{h}}_{\mathrm{h}}^{\infty} \stackrel{\infty}{\mathrm{k}}_{\mathrm{k}} \mathrm{a}_{\mathrm{hk}} \mathrm{x}_{\mathrm{hk}}\right| \leq \mathfrak{N C}_{\mathrm{A}} \mathrm{M}_{\mathrm{X}}$ qualunque siano $i$ numeri naturali $\mathrm{m}$, $\mathrm{n}$ e gli 
$\mathrm{mn}$ numeri $\mathrm{x}_{\mathrm{h}, \mathrm{k}}, \mathrm{h}=1,2, \ldots, \mathrm{m} ; \mathrm{k}=1,2, \ldots, \mathrm{n}, \mathrm{M}_{\mathrm{X}}$ essendo l'estr. sup. della matrice $\left\|\mathrm{x}_{\mathrm{hk}}\right\|$.

Abbiamo in tal modo ottenuto una caratterizzazione degli spazi $\Sigma^{*}$ e $\Sigma^{* \prime}$, non però di tipo intrinseco.

Qui di seguito vogliamo provare come, limitandoci a matrici $A$ simmetriche, si possa dare una caratterizzazione intrinseca particolarmente semplice.

Allo scopo premettiamo la seguente proposizione:

VII. Se A appartiene a $\Sigma^{* \prime}$, allora anche AB e BA vi appartengono, essendo $\mathrm{B}$ una-matrice limitata qualsiasi, e si ha $\mathrm{AB} \cdot \mathrm{X}=\mathrm{A} \cdot \mathrm{X} \overline{\mathrm{B}}, \mathrm{BA} \cdot \mathrm{X}=\overline{\mathrm{A}} \cdot \overline{\mathrm{X}} \mathrm{B}$ (il soprasegno indicando l'operazione di trasposiaione).

Anzitutto rileviamo che, pur facendo la dimostrazione pensando $X$ completamente continua, l'affermazione è valida anche se $X$ si suppone solo limitata $e$ se si sostituisce $\Sigma^{* \prime}$ con $\Sigma^{*}$, dato che è stato ultimamente provato che $\Sigma^{*} \equiv \Sigma^{* *}$.

Si ha

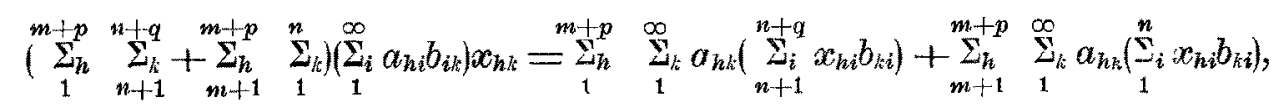

invertendo l'ordine delle sommazioni e scambiando $k$ con $i$. Ora $\left\|\sum_{n+1}^{n+q} x_{k i} b_{k i}\right\|$ è la matrice prodotto

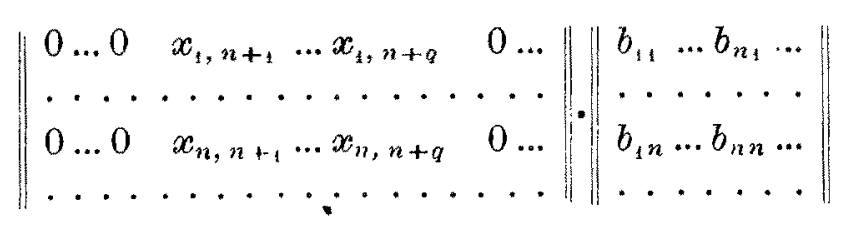

il cui estr. sup. non supera $M_{B} \cdot M_{X^{(n)}}$, indicando con $X^{(n)}$ la matrice ottenuta dalla $X$ sopprimendo le prime $n$ colonne. Ma, per una proprietà delle matrici completamente continue, è

$$
\lim _{n \rightarrow \infty} M_{X^{(n)}}=0
$$

onde, preso a piacere un numero positivo $\varepsilon$, sarà

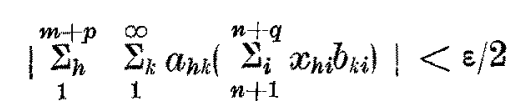

non appena si prenda $n$ abbastanza grande, qualunque siano $q, m, p$. Fissato un tale $n$, si potrà poi scegliere $m$ in modo che, qualunque sia $p$, riesca

$$
\left|\sum_{m+1}^{m+p} \sum_{i}^{\infty} \sum_{i}^{\infty} a_{h k}\left(\sum_{1}^{n} x_{h i} b_{h i}\right)\right|<\varepsilon / 2
$$


per convincersene basta tener presente che $\left\|\stackrel{n}{\Sigma}_{1}^{i} x_{h i} b_{k i}\right\|$ è la matrice comple. tamente continua

$$
\left\|\begin{array}{cccc}
x_{11} & \ldots & x_{1 n} & \ldots \\
\cdots & \ldots & \cdots & \cdots \\
x_{n 1} & \ldots & x_{n n} & \ldots \\
\ldots & \ldots & \ldots & \cdots
\end{array}\right\| \cdot\left\|\begin{array}{cccc}
b_{11} & \ldots & b_{n 1} & \ldots \\
\ldots & \ldots & \ldots & \ldots \\
b_{1 n} & \ldots & b_{n n} & \ldots \\
0 & \ldots & 0 & \ldots \\
\ldots & \ldots & \ldots
\end{array}\right\|
$$

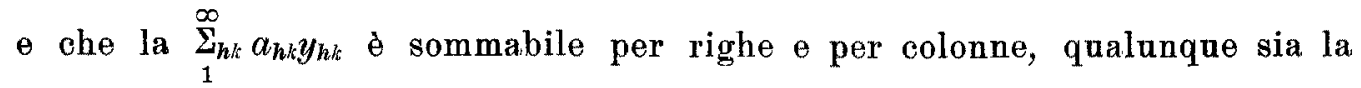
matrice limitata $\left\|y_{h i}\right\|$.

Pertanto, per $m$ ed $n$ abbastanza grandi e qualunque siano $p$ e $q$, sarà

$$
\left|\left(\underset{1}{\stackrel{m+p}{\sum_{h}}} \underset{n+1}{\sum_{n+1}^{n+q}}+\underset{m+1}{\sum_{h}+p} \underset{i}{\sum_{k}^{n}}\right)\left(\underset{1}{\Sigma_{i}} a_{h i} b_{i k}\right) x_{h k}\right|<\varepsilon
$$

ossia: se $A$ gode della proprietà specificata, della stessa proprietá godrà la matrice $A B$, essendo $B$ una arbitraria matrice limitata, e sarà $A B \cdot X=A \cdot X \bar{B}$.

Analogamente, essendo

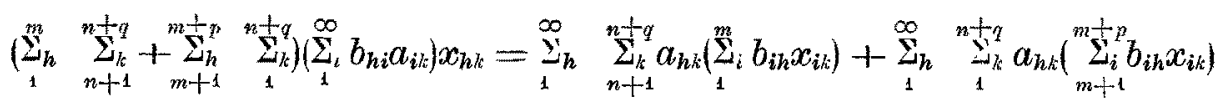

si ha un risultato analogo a quello or ora conseguito, parlando di matrice $B A$ anzichè $A B$, e sarà

$$
B A \cdot X=\overline{B A} \cdot \bar{X}=\bar{A} \bar{B} \cdot \bar{X}=\bar{A} \cdot \bar{X} B
$$

(è ovvio che se $A$ appartiene a $\mathbb{\Sigma}^{* \prime}\left(\equiv \Sigma^{*}\right.$ ), allora vi appartiene anche $\bar{A}$ ).

Ciò premesso, si può rapidamente provare la seguente proposizione:

VIII. Condizione necessaria e sufficiente affinchè la matrice simmetrica A appartenga $a \Sigma^{*}\left(\equiv \Sigma^{* \prime}\right)$ è che, detti $\rho_{1}, \rho_{2}, \ldots, \rho_{n}, \ldots$ gli autovalori di $\mathrm{A}$, sia convergente la serie $\sum_{n}^{\infty}\left|\rho_{n}\right|$.

Sia infatti $P$ la matrice ortogonale per cui

$$
\begin{aligned}
& P A \bar{P} \equiv\left\|\begin{array}{cccc}
\rho_{1} & 0 & 0 & \cdots \\
0 & \rho_{2} & 0 & \cdots \\
0 & 0 & \rho_{3} & \cdots \\
\ldots & \cdots & \cdots
\end{array}\right\|\left({ }^{6}\right)
\end{aligned}
$$

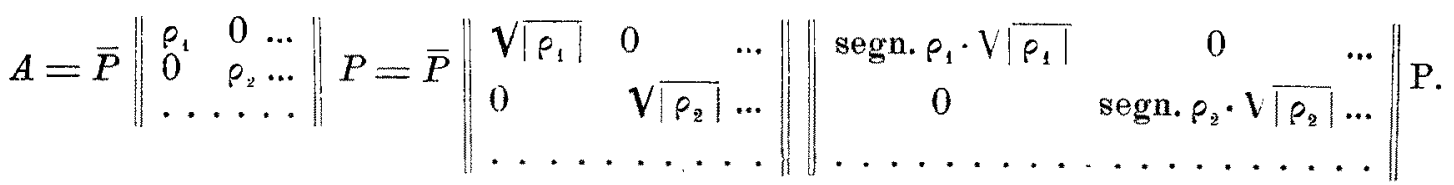

(9) Cfr. p. es. F. RIESZ, 1. c. in (1), cap. $V$; in particolare n. 100. 
Pertanto, se $\stackrel{\infty}{\Sigma}_{n}\left|p_{n}\right|$ è convergente, la matrice prodotto delle prime due all'ultimo membro nella precedente e la matrice prodotto delle ultime due, saranno ciascuna con la serie doppia dei quadrati dei termini convergente; infatti se $\left\|\alpha_{h k}\right\|$ è una matrice solo limitata $e\left\|\beta_{h k}\right\|$ è una matrice con la serie doppia dei quadrati dei termini convergente, allora le matrici $\left\|\sum_{i}^{\infty} \alpha_{h i} \beta_{i k}\right\|$ e $\left\|\sum_{i}^{\infty} \beta_{h i} \alpha_{i k}\right\|$ sono anch'esse di quest'ultimo tipo perchè si ha, p. es.

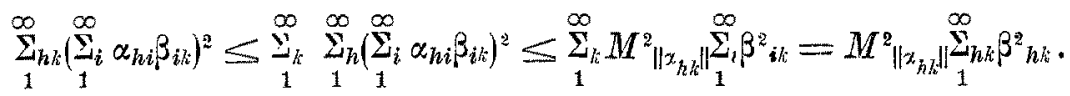

Pertanto è provata la sufficienza, se si tiene presente la II.

Per quanto riguarda la necessità, basta osservare che se $A$ appartiene a $\Sigma^{*}\left(\equiv \Sigma^{*}\right)$, allora, in forza di VII, anche $P A \bar{P}$ deve appartenere a tale

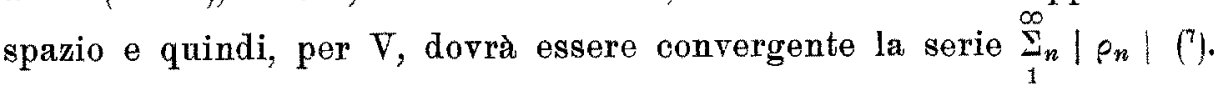

Vogliamo infine fare aleune precisazioni sul numero. $\mathfrak{K K}_{A}$, allo scopo di provare una certa proprietà di continuità del funzionale $A \cdot X$.

Fissata una matrice $A$ di $\Sigma^{*}\left(\equiv \Sigma^{*}\right)$, indichiamo con $\mathrm{A}_{\psi}{ }^{(1)}$ e $\mathrm{A}_{v}{ }^{\left({ }^{2}\right)}$ le matrici che si ottengono da A sostituendo con degli zeri tutti $i$ termini delle prime $\vee$ colonne e, rispettivanente, $\vee$ righe. Si ba che:

IX. Le due successioni $\left\{\mathfrak{O K}_{A_{v}(1)}{ }^{\prime},\left\{\mathscr{T K}_{A_{v}(2)}\right\}, v=1,2, \ldots\right.$, convergono monotonicamente a zero.

Faremo la dimostrazione solo per la prima di tali successioni, poichè il ragionamento è perfettamente simile per la seconda.

Intanto, per ogni matrice limitata $X$ di estremo superiore nguale ad uno e con le prime $v$ colonne tutte costituite da zeri, è $\left|\underset{1}{\sum_{h}}{\underset{\nu}{\infty} \sum_{k}}_{\infty}^{\infty} a_{h h} x_{h i}\right| \leq \mathscr{M K}_{A_{y}(1)}$ e quindi, in particulare, $\left|\underset{1}{\sum_{h}} \sum_{\nu+2}^{\infty} \sum_{k l i} a_{h k} x_{h k}\right| \leq \mathscr{N K}_{A_{\nu}^{(1)}}$ per ogni matrice $X$ di estremo superiore eguale ad uno e con le prime $v+1$ colonne tutte costituite da zeri; ora $\mathscr{T K}_{A_{y+1}^{(1)}}$ è il più piccolo numero non negativo $M$ per cui

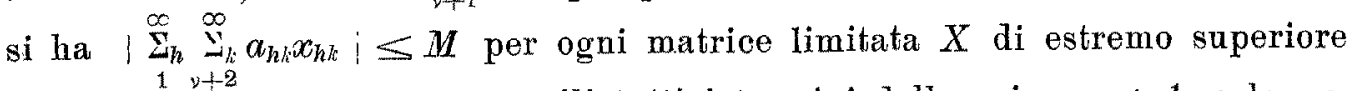
eguale ad ano, per cui sono nulli tutti i termini delle prime $\nu+1$ colonne, onde sarà

$$
\mathfrak{O K}_{A_{v}^{(1)}} \geq \mathfrak{N K}_{A_{\nu+1}^{(1)}} \text {. }
$$

Con ciò resta assodata la monotonia della successione $\left\{\mathfrak{T K}_{A_{v}(1)}\right\}$. Per provare che quest' ultima è anche convergente a zero, supponiamo, per assurdo, che esista un numero $m>0$ tale che, per ogni valore di $v$, si possa

(7) Vogliamo qui osservare come, limitatamente alle matrici $A$ simmetriche, la proposizione II si inverte, ciò̀ che il sottospazio di ᄂ*, delle matrici simmetriche, è costituito dalle matrici che si possono esprimere come prodotto di due matrici ciascuna con la serie doppia dei quadrati dei termini convergente.

E probabile che ciò valga per tutte le matrici di $\Sigma^{*}$ e non solo per quelle simmetriche. 
trovare una matrice limitata $X_{\nu}$ di estremo superiore eguale ad uno e con le prime $\vee$ colonne tutte costituite da zeri per cui riesca

e quindi anche

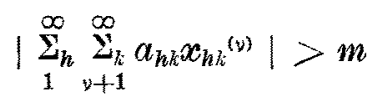

$$
\sum_{1}^{\infty}{\underset{\Sigma}{\Sigma}}_{k+1}^{\infty} a_{h k} x_{h k}^{(v)}>m
$$

previo eventuale cambiamento di segno a tutti i termini di una o più colonne di tale matrice, operazione che noi pensiamo già effettuata quando indichiamo la matrice $\operatorname{con} X_{\nu}$.

Ora, trattandosi di serie doppie convergenti e sommabili per righe e per colonne, per ovvie ragioni di convergenza e per l'ipotesi fatta, si potrà tro. vare una successione crescente di numeri interi $\nu_{1}, \gamma_{2}, \ldots$ e una successione di matrici limitate $X^{(0)}, X^{(1)}, \ldots$, con

$$
X^{(r)} \equiv\left\|\begin{array}{ccc}
0 \ldots x_{1,{ }_{r}+1}^{(r)} \ldots x_{1,{ }_{r+1}}^{(r)} & 0 \ldots \\
\ldots \ldots \ldots \ldots
\end{array}\right\| \quad \quad\left(v_{0}=0\right)
$$

ciascuna di estremo superiore non maggiore di uno, per cui sia

$$
\sum_{\Sigma_{h}}^{\infty} \sum_{y_{k}+1}^{y_{r}+1} a_{k k} x_{k k}{ }^{(n)}>m \quad r=0,1, \ldots
$$

Proviamo che se $\rho_{0}, \rho_{1}, \ldots$ è un'arbitraria successione di numeri reali per cui $\Sigma_{h}^{\infty} \rho^{2}{ }_{h}$ è convergente, allora le ridotte della serie $\rho_{0} X^{(0)}+\rho_{1} X^{(1)}+\ldots+$ $+\rho_{n} X^{(n)}+\ldots$, convergono fortemente, onde la matrice limite

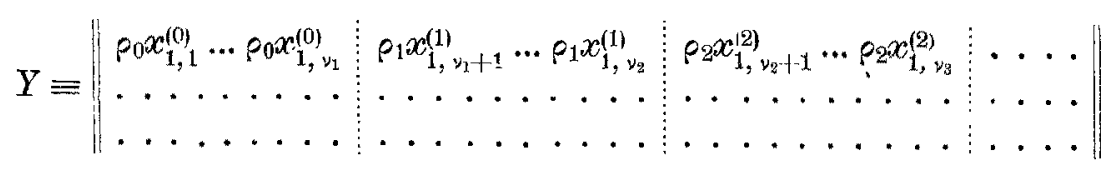

sard limitata (anzi addirittura completamente continua essendo tali le ridotte considerate), e inoltre di estremo superiore non maggiore di $\sqrt{\sum_{h}^{\infty} \rho^{2} h}$. Infatti se $\left\|\omega_{h k}\right\|$ è una qualsiasi matrice prodotto di due matrici $\left\|\alpha_{h k}\right\|$ e $\left\|\beta_{h k}\right\|$ $\operatorname{con} \sum_{1}^{\infty} \alpha_{h k}$ e $\sum_{1}^{\infty} \beta_{h k} \beta_{h k}^{z}$ convergenti, si ha

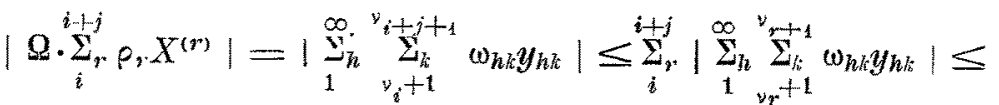

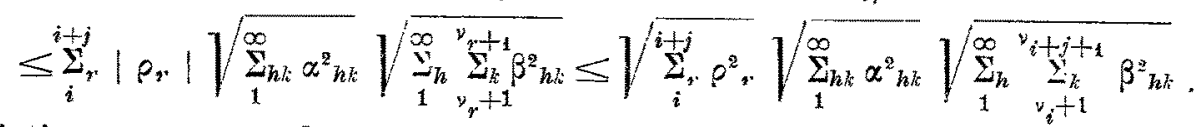

se si tiene presente che

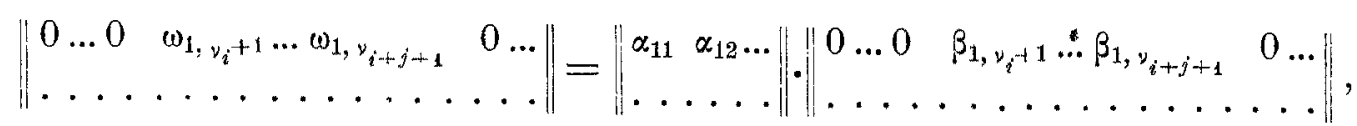


e la diseguaglianza di Lagrange-Cadohr. Pertanto non appena $i$ è abbastanza grande, e qualunque sia $j$, sara, per l'osservazione in fine di $I$,

$$
M_{\substack{\Sigma_{r} \rho_{r} X^{(r)} \\ M_{i}}} \leq \sqrt{\sum_{i, \rho^{2}{ }_{r}}^{i+j}}<\varepsilon,
$$

essendo $\varepsilon$ una quantità positiva fissata a piacere. essendo $\varepsilon$ una quantita positiva tissata a piacere.
Ora, se si suppone che i numeri $\rho_{0}, \rho_{1}, \rho_{2}, \ldots$ siano tutti positivi e $\stackrel{\infty}{*}_{0}^{\infty} \rho_{h}$
sia divergente, si ha, da una parte,

e dall'altra, per la (5),

$$
\left|\sum_{1}^{\infty} a_{h k} y_{n k l}\right| \leq \Re_{A} \sqrt{\sum_{0}^{\infty} \rho^{2}{ }_{r}},
$$

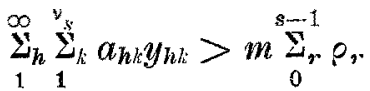

$$
\begin{aligned}
& s=1,2, \ldots,
\end{aligned}
$$

manifestamente contradditorie.

Da quanto abbiamo ora provato discende immediatamente l'annunciata proprietà di continuità del funzionale $A \cdot X$. Precisamente:

$\mathrm{X}$. Se $\left\{\mathrm{X}^{(y)}\right\}$ è una successione di matrici limitate semplicementé convergente alla matrice limitata $\mathrm{X}$, allora si ha $\lim _{\nu \rightarrow \infty}\left(\mathrm{A} \cdot \mathrm{X}^{(\nu)}\right)=\mathrm{A} \cdot \mathrm{X}$.

Infatti

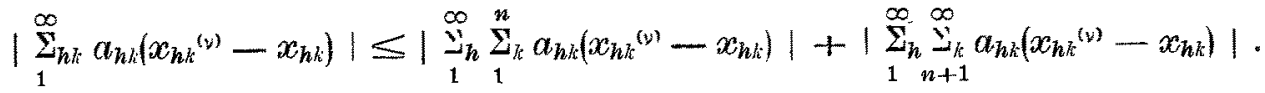

Ora $\lim _{v \rightarrow \infty} \sum_{h}^{\infty} a_{h i}\left(x_{h k}^{(v)}-x_{h k}\right)=0$ per una proprietà del prodotto scalare di di due successioni di $H$-vettori, l'una convergente fortemente e l'altra convergente semplicemente (a zero). D'altra parte sarà per un opportuno $M_{\text {a }}$ $M_{X^{(y)}<M}$ qualunque sia $\gamma$, onde il secondo termine a secondo membro nella precedente riesce maggiorato da

$$
29 \mathcal{K}_{A_{n}^{(1)}} \cdot M
$$

e di qui segue subito l'asserto in base a IX.

Vogliamo, a questo proposito, rilevare esplicitamente come la proprietì ora provata non rientra nella proprietà, generale, di continuità di un funzionale lineare limitato $\left.{ }^{8}\right)$. Precisamente, in base alla metrica accennata nell'introduzione e a III $\left(\right.$ III $\left.^{\prime}\right)$ sarà in $\Sigma^{\prime}(\Sigma) \lim _{\nu \rightarrow \infty}\left(A \cdot X^{(\nu)}\right)=A \cdot X$ se $\lim _{\nu \rightarrow \infty} M_{X-X^{(\nu)}}=0$, ciò che assicura la continuità del funzionale $A \cdot X$ se la successione $\left\{X^{(y)}\right\}$ di matrici di $Y^{\prime}(\Sigma)$ converge fortemente alla matrice $X$.

La $X$ invece assicura la continuità del funzionale $A \cdot X$ anche relativa. mente a successioni solo semplicemente convergenti di matrici di $\Sigma^{\prime}(\Sigma)$.

(8) Cfr. p. es. G. Ascoli, Sugli spazi lineari metrici e le loro varietà lineari, I, "An. nali di Matematica', 1932 , pp. $400-46$. 